\title{
Measurement setup for the determination of the nonlinear refractive index of thin films with high nonlinearity
}

Steinecke, Morten, Kellermann, Tarik, Jupé, Marco, Ristau, Detlev, Jensen, Lars

Morten Steinecke, Tarik Kellermann, Marco Jupé, Detlev Ristau, Lars Jensen, "Measurement setup for the determination of the nonlinear refractive index of thin films with high nonlinearity," Proc. SPIE 10805, Laser-Induced Damage in Optical Materials 2018: 50th Anniversary Conference, 1080524 (20 November 2018); doi: 10.1117/12.2500341

SPIE. Event: SPIE Laser Damage, 2018, Boulder, Colorado, United States 


\title{
Measurement setup for the determination of the nonlinear refractive index of thin films with high nonlinearity
}

\author{
Morten Steinecke*a, ${ }^{*}$ arik Kellermann ${ }^{\mathrm{a}}{ }$ Marco Jupé $^{\mathrm{a}}$, Detlev Ristau ${ }^{\mathrm{a}, \mathrm{b}}$, Lars Jensen ${ }^{\mathrm{a}}$ \\ ${ }^{a}$ Laser Zentrum Hannover e.V., Laser Components Department, Hollerithallee 8, 30419 Hannover, \\ Germany; \\ ${ }^{\mathrm{b}}$ Institut for Quantum Optics, QUEST, Leibniz Universität Hannover, Germany
}

\begin{abstract}
The exploitation of nonlinear effects in multi-layer thin films allows for optics with novel functions, such as all- optical switching and frequency conversion. In this contribution, an improved interferometric setup for the measurement of the nonlinear refractive index in dielectric substrates and deposited single layers is presented. The setup is based on the wave front deformation caused by the self-focusing in the measured samples. Additionally, measurement results for a highly nonlinear material, indium-tin-oxide (ITO) are presented with respect to the materials power handling capabilities and compared to values from other materials.
\end{abstract}

Keywords: Kerr-effect, material science, self-focusing, thin films, nonlinear optics

\section{INTRODUCTION}

The development of optical thin films has reached a high technical level. Optical thin film systems can handle complex demands regarding spectral characteristics, power handling capability and phase modification. Nevertheless, the requirements on optical components are also growing very fast, and the necessary development in the thin film manufacturing is presenting itself to be increasingly difficult $[1,2]$. Especially ultra-short-pulsed laser applications require a detailed understanding of the material properties, linear as well as non-linear, of coating and substrate materials. The destructive processes are usually investigated with particular attention, but the non-destructive processes below the laser induced damage threshold also influence the properties of dielectric films significantly [3]. This opens up possibilities to create optical components with new functionalities by exploiting nonlinear effects below the damage threshold. However, to achieve this, materials with suitable nonlinear properties and a precise knowledge of these properties are necessary. As the optical properties of thin films can differ significantly from those of corresponding bulk material [4], it is necessary to achieve direct knowledge of the layer materials used for each application. Special attention has to be payed to the power handling capabilities in relation to the nonlinear refractive index of the material, as a strong nonlinear response below the damage threshold of the material is desirable.

To characterize these material properties, a special measurement procedure was previously developed and qualified to measure the nonlinear refractive index $\left(\mathrm{n}_{2}\right)$ for bulk substrates as well as deposited thin films [5]. The procedure is based on a combination of the established z-scan method [6], and a Mach-Zehnder-interferometer [7]. In the classical z-scan method, the sample is moved along a focused beam, and the self-focusing in the sample is translated into an intensity variation on a detector. The combination with a Mach-Zehnder-interferometer allows, by providing an undisturbed reference beam, the monitoring of the wave front deformation caused by the self-focusing in the material, potentially improving the sensitivity of the instrument. The gained data of the wave front curvature variation is fitted applying a beam propagation model based on the optical matrix formalism with modifications to account for the optical Kerr-effect.

For the characterization of the thin film material, a single layer of ten micrometer is manufactured using an Ion-BeamSputtering process. Appling the introduced measurement setup, the manufactured sample is characterized regarding the nonlinear refractive index as well as the power handling capabilities of the material. The determined material properties are evaluated and compared to other optical materials as well as values from literature.

*m.steinecke@lzh.de; $\quad$ phone $\quad$ +49511-2788-297; $\quad$ fax $\quad+49511-2788-100 ; \quad$ www.lzh.de 


\section{THE KERR-EFFECT IN OPTICAL MEDIA}

One of the effects caused by the third order nonlinear polarization is the optical Kerr-effect. Similar to the classical electrooptical Kerr-effect, where an external electric field is applied to an optical medium for changing the refractive index and inducing birefringence, the optical Kerr-effect changes the refractive index of the optical medium. Instead of requiring an external field though, in the case of the optical Kerr-effect, the electromagnetic radiation incident on the optical medium is itself responsible for the refractive index change [8]. The classical refractive index $n$ of the optical medium then splits up into two parts, a constant 'linear' index $n_{0}$ and a varying, nonlinear index $n_{\mathrm{NL}}$. The so induced change in the refractive index is proportional to the square of the electric field of the incident radiation, and consequently to its intensity. The factor that relates the refractive index change with the applied radiation intensity is called the nonlinear refractive index and is typically denoted as $\mathrm{n}_{2}$ :

$$
n=n_{0}+n_{\mathrm{NL}}=n_{0}+n_{2} * I
$$

When a laser beam of the necessary intensity propagates through an optical medium, this leads to a refractive index change in the medium which is proportional to the beam's spatial intensity distribution. The typical laser beam one would expect in the laboratory however does not have a spatially homogenous intensity profile, but rather a circular symmetrically shape with a maximum at the beams center, e.g. a Gaussian $\mathrm{TEM}_{00}$-mode. This leads to a refractive index profile with the undisturbed refractive index $\mathrm{n}_{0}$ at the beams edges, where the intensity is low, and typically a maximum ( $\mathrm{n}_{2}>0$ for typical optical media), at the beam's center. This type of refractive index profile causes a phase shift similar to that of a thin lens, which in turn causes a focusing of the optical beam in the medium, the so called Kerr-self-focusing. For a medium which is thin in comparison with the Rayleigh-length of the laser beam, the focal length $f_{\text {Kerr }}$ of the so called Kerr-lens induced by the laser beam is given by:

$$
f_{\text {Kerr }}=\frac{a \pi w^{4}}{4 n_{2} d P}
$$

Hereby, is $P$ the power of the incident beam, $d$ the geometric length of the beam's path through the medium and $w$ the beam radius of the beam at the position of the optical medium. $a$ is a correction factor (usually $a>1$ ) which is necessary to compensate the difference between the actual, in this case Gaussian beam shape, and a parabolic beam profile [9].

The self-focusing of an optical beam can be a cause of laser induced damage [10] and is often used as a way to measure the nonlinear refractive index of optical materials, as presented in the next section.

\section{INTERFEROMETRIC SETUP FOR MEASUREMENTS OF THE NONLINEAR REFRACTIVE INDEX}

\subsection{Classical z-scan}

An established method for determination of the nonlinear refractive index is the so-called z-scan [11]. In this method, the sample is moved along the path of a focused beam to vary the intensity on the sample. This causes focusing at different positions of the sample and changes the beam's diameter in the far-field. This change in diameter is monitored by measuring the power transmitted through an aperture, which varies according to the beams size before the aperture. Figure 1 shows a schematic setup of a z-scan measurement and figure 2 the typical shape of the resulting signal. 


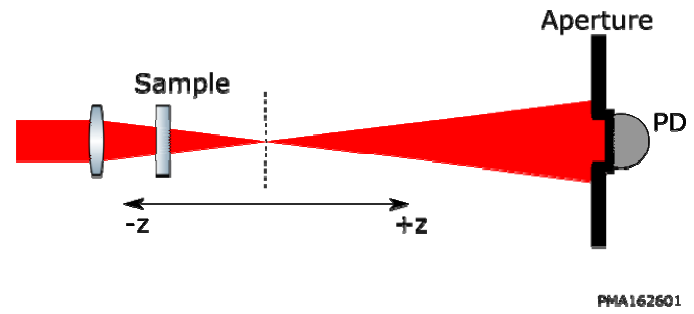

Figure 1.: Principle of the z-scan setup. The incoming laser beam induces a Kerr-lens in the sample, which is moved along the beam's path to create varying focal lengths. This causes the beam diameter on the aperture to change, which consequently changes the power detected in the photodetector (PD) (schematically taken from [12]).

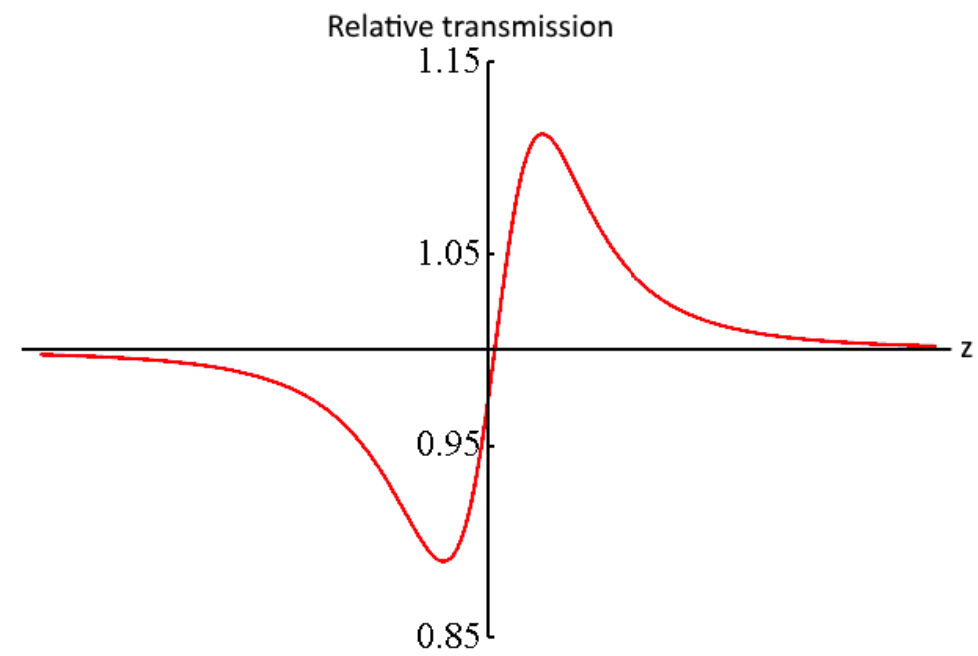

Figure 2.: Typical signal shape for a z-scan measurement with the sample's $\mathrm{n}_{2}>0$. When the sample is in front of the beams focus point, the detected power on the photodetector is lower and contrary, after the sample is behind the focal point, the power detected rises due to an increased beam diameter. The power is referenced to the undisturbed transmission.

The z-scan shows the response of one beam parameter to the optical Kerr-effect, yet a Gaussian beam is completely described by its complex beam parameter, which consists of two real parameters, the beam's radius and the wave front curvature. The wave front curvature has a much higher dynamic range, with typical curvature radii ranging from centimeters at the Rayleigh length up to infinity at focus locations. This, in principle, would enable a more sensitive measurement of the focusing effects. Additionally, the z-scan is sensitive to absorption effects in the sample, where especially the nonlinear absorption is able to deform the typical signal shape significantly [11].

\subsection{Interferometric measurement}

To measure the wave front curvature of an optical beam, interferometric methods are well established [13]. In this contribution a Mach-Zehnder-interferometer is used, where the incident laser radiation is propagating in two arms. One arm provides a reference beam with flat wave front, and the other arm contains the sample, which is, similarly to the classical z-scan, moved along a focused beam. The beam splitters are adapted to this special setup and deliver the necessary power for the sample beam. After the beam has passed the sample, it is collimated again to achieve a constant beam diameter. The reference and sample beam are then superimposed at the second beam splitter and the resulting interference pattern is recorded by a commercial "Basler pulse" camera. Figure 3 shows the schematic layout of the setup. 


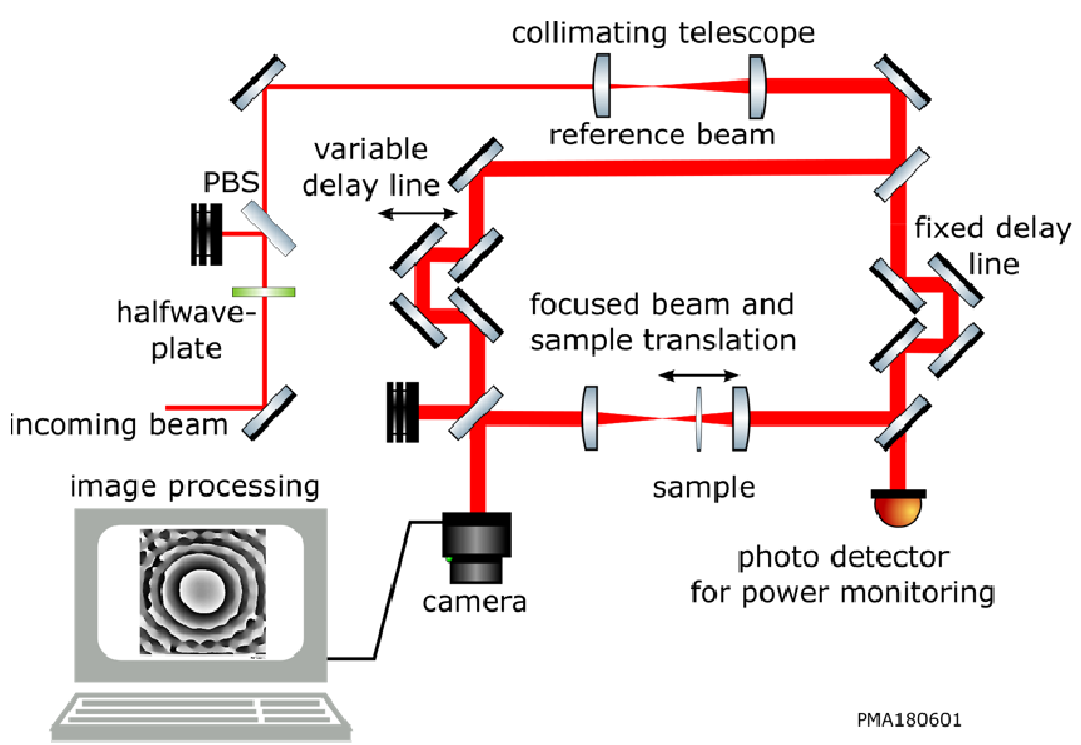

Figure 3.: A schematic layout of the interferometric setup for the measurements of the nonlinear refractive index. The incoming laser beam is attenuated to the desired power level, and then collimated in a first telescope. The beam is propagating in the two arms of the interferometer. The beam splitters are chosen in such a way, that $90 \%$ of the laser power is transmitted into the sample-arm and $10 \%$ is reflected to serve as reference beam. The reference-beam provides a nearly plane reference wave front, while the beam passing through the other arm is "disturbed" by the sample. The delay lines ensure a path length difference below the coherence length of the utilized laser-radiation.

The measurements were performed with a mode locked, $\mathrm{Nd}: \mathrm{YVO}_{4}$ "Lumera Rapid"-laser system with a 12ps pulse duration and a repetition rate of $10 \mathrm{kHz}$. Additionally, a commercial, external, single-pass "NeoLase"-amplifier was used to increase the available pulse energies to up to $100 \mu \mathrm{J}$.

The sample measured in this publication consists of a $10 \mu \mathrm{m}$ thick ITO-layer which was deposited on a $1 \mathrm{~mm}$ fused silica substrate. The layer was manufactured applying an Ion Beam Sputtering (IBS) coating process with a pre-mixed ITOtarget. The gas used for sputtering was argon and additional oxygen was supplied during the coating.

\subsection{Retrieving the nonlinear refractive index}

The measurement procedure and the evaluation with a modified optical matrix formulism are described in detail in [5]. In this publication, only a brief overview is given.

The recorded interference patterns are processed with the so called "linear-carrier-method" to retrieve the information about the shape of the wave front. The method itself is given in detail in [13], here only a short summary will be presented. To use the "linear-carrier-method", the two interfering beams have to be superimposed under a small angle, to achieve a stripe-like pattern. This stripe pattern contains the information about the phase distribution of the sample beam. To obtain this phase distribution, a two-dimensional Fast-Fourier-Transformation (2D-FFT) is performed to calculate the spatial frequencies contained in the interference pattern. In this frequency image, the pattern formed by the stripes is visible as two bright spots point-symmetrical to the center of the spectrum. Figure 4 shows an example for the stripe-link interference pattern and the 2D-spectrum. One of these spots is then isolated and placed in the center of a new spectrum of equal size. This new spectrum is then back-transformed with an inverse 2D-FFT. The distribution of the complex phase of the resulting image is then equal of the phase distribution of the sample beam. A typical phase profile measured with this procedure is presented in figure 5. The jumps in the image result from an arctangent applied to calculate the complex phase and need to be compensated for determination of the wave front curvature. For ease of calculation, this is done by taking $1 \mathrm{D}$ line-scans from the 2D phase distribution rather than directly in the 2D image. A circular fit is applied to the compensated phase front to determine the Radius of Curvature (ROC). Figure 5 schematically shows the lines taken from the 2D image. To obtain the nonlinear refractive index from the measured radii, the measurement procedure is simulated with the optical matrix formalism, where each section of the beam propagation is modeled with the corresponding matrices. The self-focusing in the sample is calculated as a sequence of lenses with small stretches of the 
sample medium in between (so called "distributed-lens-approximation") [14]. This allows the calculation of the beam parameters at the location of the camera and therefore the comparison with the measured values. Figure 6 shows the simulated variation of the ROC for a $1 \mathrm{~mm}$ quartz sample with and without a highly nonlinear layer deposited on one side.

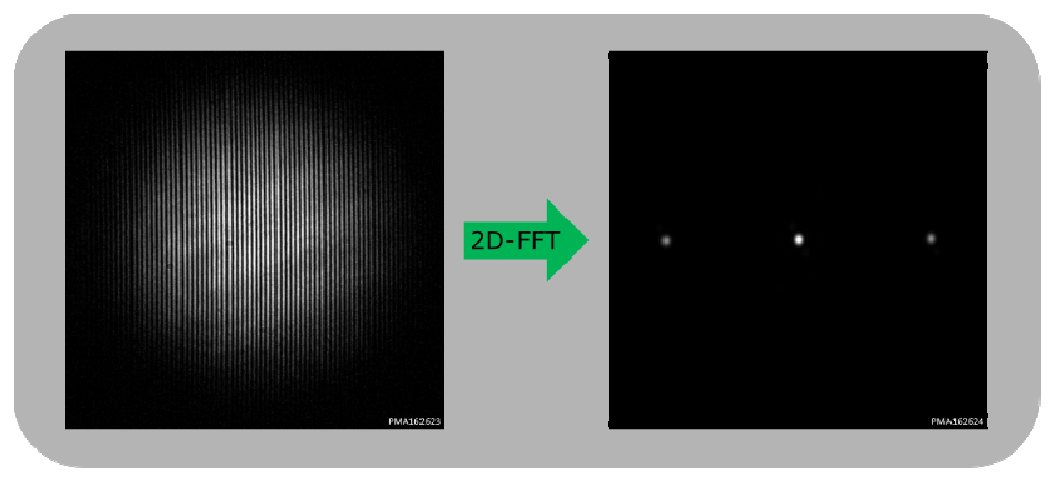

Figure 4.: An exemplary interference pattern and the resulting 2D-spectrum of spatial frequencies. The stripe-like pattern of the interference pattern is represented by the symmetrical maxima in the spectrum.

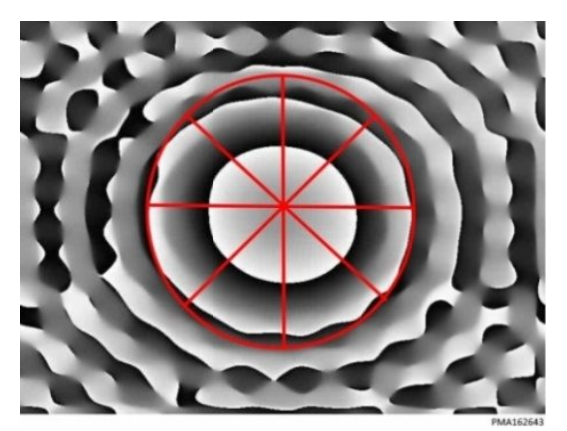

Figure 5.: A typical phase distribution reconstructed from the interference pattern. The jumps from bright to dark are produced during the reconstruction and need to be compensated. This is done by scanning discrete lines and compensating the phase-jumps for each line separately.

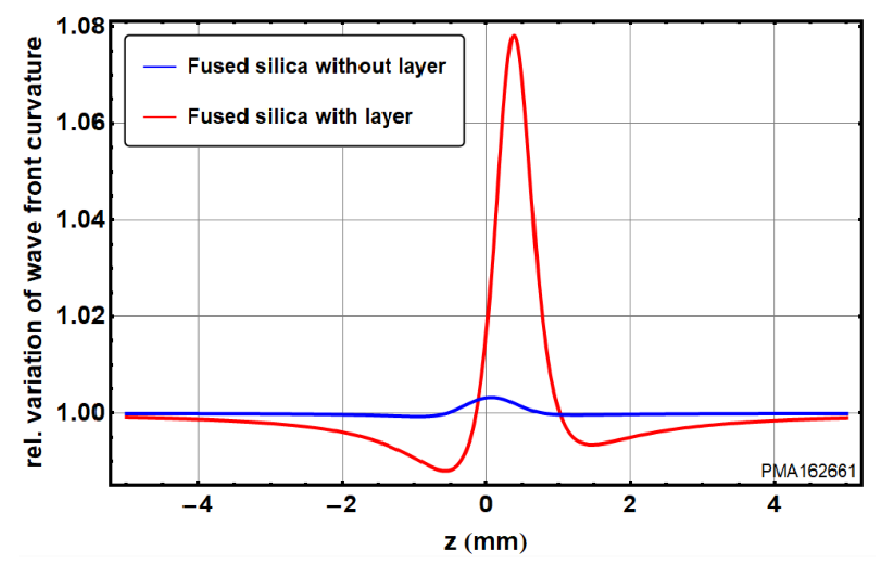

Figure 6.: Simulations for a $(1 \mathrm{~mm})$ fused silica sample with and without a layer deposited on one side. The curves show a clear increase caused by the layer. In the simulations presented here, the nonlinear refractive index of the layer is a factor of 1000 higher than that of the substrate, and the layer thickness is $10 \mu \mathrm{m}$. 


\section{MEASUREMENT RESULTS}

\subsection{Measurements of the nonlinear refractive index}

The measurements were performed with the setup described in section 3 . The measured variation of the changing ROC and the fitted curve are presented in figure 7 . The pulse energy used for the presented measurement was $2.45 \mu \mathrm{J}$, and the relative values presented referenced to a measurement with $0.04 \mu \mathrm{J}$. The determined nonlinear refractive index is $2.3 * 10^{-13} \mathrm{~cm}^{2} / W$, and therefore almost three orders of magnitude higher than the corresponding value of the substrate which is approximately $3 * 10^{-16} \mathrm{~cm}^{2} / W$ [15]. Values for a variation of optical substrates measured with the interferometric setup are, together with corresponding literature values, presented in table 1 .

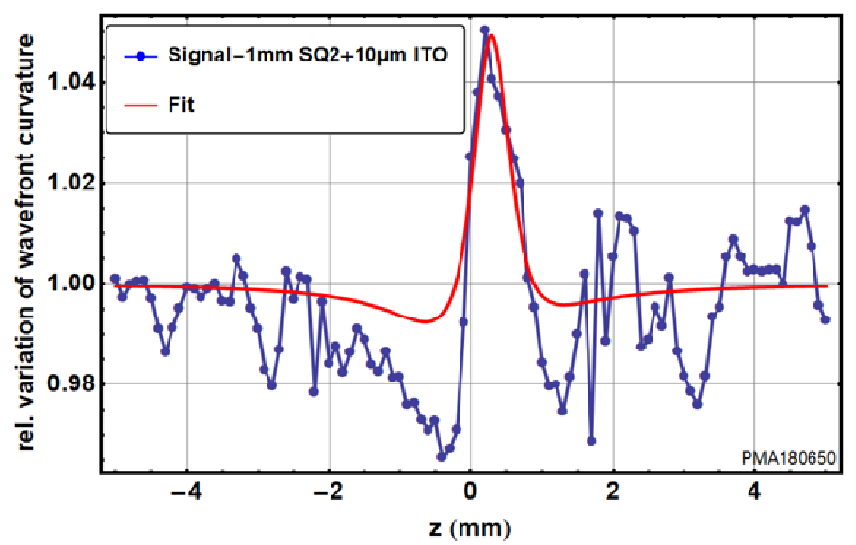

Figure 7.: The relative change of wave front curvature induced in the ITO-sample. The blue graph shows the measured values and the red graph represents the fitting curve obtained from the matrix formulism.

Table 1: Measured nonlinear refractive indices of optical substrate materials [5] and values from literature for comparison.

\begin{tabular}{|c|c|c|c|c|}
\hline Material & Thickness $(\mathrm{mm})$ & $\mathrm{n}_{0} @ 1064 \mathrm{~nm}$ & $\mathrm{n}_{2} \cdot\left(10^{-16} \mathrm{~cm}^{2} / \mathrm{W}\right)$ & $\mathrm{n}_{2}$ lit $\left(10^{-16} \mathrm{~cm}^{2} / \mathrm{W}\right)$ \\
\hline SQ2 & 2 & 1.4496 & 4.3 & $1.9-3[15]$ \\
\hline SQ2 & 6.35 & 1.4496 & 3.8 & $1.9-3[15]$ \\
\hline YAG (@1300) & 2.6 & 1.8147 & 7.1 & $7.7[16]$ \\
\hline B270 Glas (@620) & 2 & 1.5186 & 3.3 & $3.4[17]$ \\
\hline
\end{tabular}

\subsection{Measurements of the power handling capability}

In addition to the nonlinear refractive index, also the power handling capability of the ITO-layer was evaluated in the same setup. To estimate the LIDT of the material, the scanning procedure was repeated at increasing power levels, until the wave front curvature changed drastically, i.e. the material was destroyed at the location of the laser beam. This change as well as the destroyed spot is illustrated in figure 8. The destruction occurred at a pulse-energy of $4.1 \mu \mathrm{J}$, which equals a fluence of $1.33 \mathrm{~J} / \mathrm{cm}^{2}$. Additionally, in figure 9 the fluence necessary for destruction of the sample is compared to the fluence at which the nonlinear refractive index was measured. It is observed, that the nonlinear effect occurs already well below the damage threshold limits of the sample. 


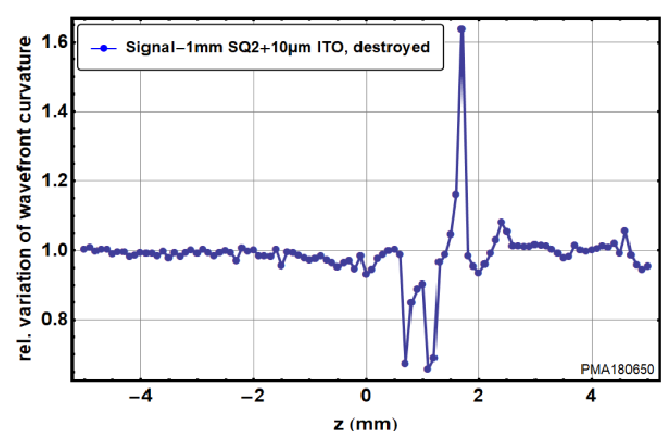

(a)

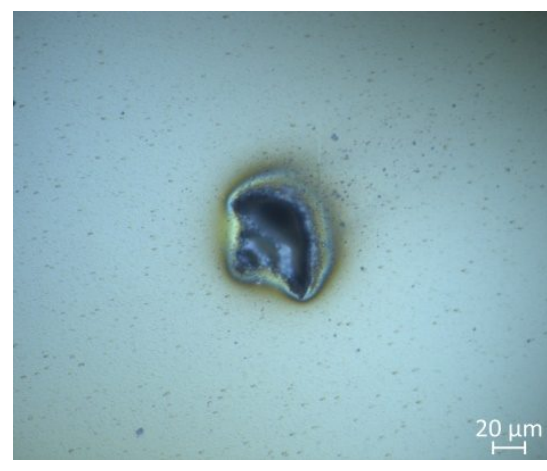

(b)

Figure 8.: (a) the measured relative variation of the ROC during the laser induced destruction of the sample. The destruction occurred at a fluence of $1.33 \mathrm{~J} / \mathrm{cm}^{2}$. The ROC variation caused by the destruction of the sample is much higher than the one caused during the measurement of the nonlinear refractive index. (b) shows destruction at the damaged test site caused by intensities at the threshold.

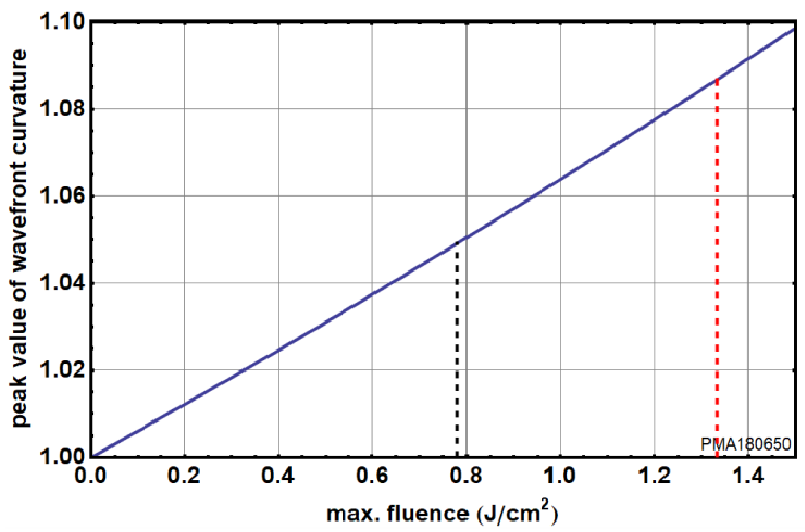

Figure 9.: The fluence applied for measuring the nonlinear refractive index is compared to the damage threshold of the sample. To estimate the result of increasing the power used for the measurement, the expected peak variation of the ROC is calculated.

\section{SUMMARY}

In this work, a wave front based method for measuring the nonlinear refractive index of optical substrates and thin film materials is presented and evaluated. The method is based on the well-established z-scan approach, instead of monitoring the power of the transmitted beam, the wave front curvature is monitored. The measurement process is then applied to the investigation of the highly nonlinear material, ITO. To measure the nonlinear refractive index of ITO-layers for later use in optical thin film stacks, an ITO-layer was deposited using an Ion-Beam-Sputtering process. The $\mathrm{n}_{2}$ of the manufactured layer was determined to be $2.3 * 10^{-13} \mathrm{~cm}^{2} / W$, which agrees with the value from literature, which states an $\mathrm{n}_{2}$ of $5 * 10^{-14} \mathrm{~cm}^{2} / W$ for $970 \mathrm{~nm}[17]$ and predict an increasing $\mathrm{n}_{2}$ towards longer wavelengths. Finally, the power handling capability of the sample was compared to the power necessary to measure the nonlinear properties. It was shown that the sample exhibits strong nonlinear properties while the laser intensity stays below the damage threshold. The presented results indicate that ITO-layers are a good candidate for nonlinear photonic devices consisting of thin film stacks, for example all-optical switching devices. 


\section{ACKNOWLEDGEMENTS}

The authors thank the German Federal Ministry of Education and Research (BMBF) for the financial support of the research projects "Kerr-Band-Schalter" (contract no. 13N13967) and the project "THG-Schicht" (contract no. $13 \mathrm{~N} 14063)$.

\section{REFERENCES}

[1] Pervak, V., Razskazovskaya, O., Angelov, I., Vodopyanov, K. and Trubetskov, M., "Dispersive mirror technology for ultrafast lasers in the range $220-4500 \mathrm{~nm}$. Advanced Optical Technologies." Adv. Opt. Techn. 0, 1-9. (2013)

[2] Ristau, D. [Laser Induced Damage in Optical Materials], CRC Press, Boca Raton (2014)

[3] Fedulova, E., Trubetskov, M., Amotchkina T., Fritsch K., Baum, P., Pronin, O., and Pervak, V. "Kerr effect in multilayer dielectric coatings" Opt. Express 24(19), 21802-21817 (2016)

[4] Rao, M. C. and Shekhawat, M. S. "A brief Survey on Basic Properties of thin Films for Device Application" Int. J. Mod. Phys. Conf. Ser. 22, 576 (2013).

[5] Steinecke, M., Jupé, M., Kiedroswki, K., and Ristau, D. "Measurement of the nonlinear refractive index in optical thin films", Proc. SPIE 10447, Laser-Induced Damage in Optical Materials 2017, 104472A, (22 March 2018)

[6] Sheik-Bahae, M., Said, A. A. and Stryland, E. W. V., "High-sensitivity, single-beam n2-measurements." Opt. Lett. 14(17), 955-957 (1989)

[7] Zehnder, L. "Ein neuer Interferenzrefraktor" Zeitschrift für Instrumentenkunde 11, 275-285 (1891)

[8] Boyd, R. W. [Nonlinear optics] $3^{\text {rd }}$ Ed.,, Academic Press, Cambridge, Massachusetts, 11 (2008)

[9] Vaziri, M. R. Comment on "Nonlinear refraction measurements of materials using the moiré deflectometry", Opt. Commun. 357 (2015), dec, 200-201.

[10] Solieau, M. J., Williams, W. E., Mansour, N. and Van Stryland, E. W. "Laser-induced damage and the role of self-focusing", Opt. Eng. 28(10), 281133 (1989).

[11] Yin, M., Li, H., Tang, S., Ji, W., "Determination of nonlinear absorption and refraction by single Z-scan method" Appl Phys B (2000). 70, 587-591. (2000)

[12] Powers, P. E. [Fundamentals of Nonlinear Optics], CRC Press, Boca Raton (2011)

[13] Malacara, D., Servín, M. and Malacara, Z. [Interferogram analysis for optical testing] $2^{\text {nd }}$ Ed., CRC Press, Boca Raton (2011)

[14] Sheik-Bahae, M., Said, A. A., Hagan, D. J., Soileau, M. J., and Van Stryland, E. W. "Nonlinear refraction and optical limiting” Opt. Eng., 30(8), 1228-1236. (1991)

[15] Milam, D. "Review and assessment of measured values of the nonlinear refractive-index coefficient of fused silica” Appl. Optics 37(3), 546-550 (1998)

[16] Samuel, P., Ensley, T. R., Hu, H., Hagan, D. J., Van Stryland, E. W. and Gaume, R. "Nonlinear refractive index measurement on pure and Nd doped YAG ceramic by dual arm Z-scan technique" AIP Conf. Proc. 1665, 1, 060010 (2015)

[17]Zahirul Alam, M., De Leon, I., Boyd, R. W. "Large optical nonlinearity of indium tin oxide in its epsilon-nearzero region", Science 352(6287), 795-797 (2016) 